\title{
Single-Longitudinal-Mode Pumped Pulsed-Dye Amplifier for High-Resolution Laser Spectroscopy
}

\author{
M. Verlinde, ${ }^{1, \text { a) }}$ R. Ferrer, ${ }^{1}$ A. Claessens, ${ }^{1}$ C.A. Granados, ${ }^{1,2}$ S. Kraemer, ${ }^{1}$ Yu. Kudryavtsev, ${ }^{1}$ D. Li, ${ }^{3}$ P. Van den \\ Bergh, ${ }^{1}$ P. Van Duppen, ${ }^{1}$ and E. Verstraelen ${ }^{1}$ \\ ${ }^{1)} K U$ Leuven, Instituut voor Kern- en Stralingsfysica, Celestijnenlaan 200D, 3001 Leuven, \\ Belgium \\ 2) CERN, CH-1211 Geneva 23, Switzerland \\ ${ }^{3)}$ Edgewave GmbH, Carlo-Schmid-Str. 19, 52146 Würselen, Germany
}

(Dated: 11 September 2020)

\begin{abstract}
The In-Gas-jet Laser Ionization and Spectroscopy (IGLIS) technique relies on narrow-bandwidth, high-peakpower, short-pulse-length $(\approx 10 \mathrm{~ns})$ and high-repetition-rate laser pulses to probe, precisely and efficiently, the hyperfine structure of medium-heavy and heavy isotopes, embedded in a supersonic jet. The power and repetition rate requirements of the laser system are met by combining $\approx 100 \mathrm{~W}, 8 \mathrm{~ns}$ pulse width, $10 \mathrm{kHz}$ commercial Nd:YAG pump lasers with a single-mode continuous wave (cw) seeded Pulsed Dye Amplifier (PDA). The common multi-longitudinal-mode operation of these Nd:YAG pump lasers causes, however, undesirable frequency sidebands in the output spectrum of the PDA system, hindering the attainable spectral resolution, a correct interpretation and an accurate analysis of the hyperfine spectra. In this article, a new prototype Nd:YAG laser is presented, which combined with the PDA system, is capable of providing quasi transform-limited laser pulses at $10 \mathrm{kHz}$, with only limited losses in laser power. This system reduces any spectral sideband amplitude below a proven upper limit of $0.2 \%$ with one order of magnitude extra reduction expected based on simulations. A full characterization of both the Nd:YAG and PDA laser systems is done by studying the temporal and frequency behavior in detail. The study is finalized by a performance benchmark of this combined laser system in the hyperfine spectroscopy of copper isotopes, showcasing its applicability for future IGLIS studies.
\end{abstract}

\section{INTRODUCTION}

Dye lasers remain widely used systems in the field of laser spectroscopy. Their high gain and wide tunability in a broad and continuous range of the electromagnetic spectrum $(\approx 320-900 \mathrm{~nm})$ allow for intense laser pulses to be produced, making, additionally, efficient conversion to the Ultra Violet (UV) region, or even further, possible. These pulses can then be used for many atomic and molecular laser-spectroscopy applications. Lately, the demand for narrowband dye-laser based pulses at high-repetition rate $(\sim 10 \mathrm{kHz})$ has grown. Particularly to minimize experimental duty-cycle losses and allow for high-resolution resonance ionization spectroscopy, selective optical pumping, etc. with nanosecond transformlimited laser pulses. Alongside the development of the more complex injection-locking technique with Ti:Sa cavities to produce these kind of laser pulses ${ }^{1}$, direct pulsed amplification of a continuous-wave (cw) narrowbandwidth laser source has been introduced ${ }^{2-10}$. A comprehensive overview of both laser types in light of the applications discussed here is given $i^{11}$. The pulsed dye amplifier (PDA) systems consist of several components. First, a source of single-mode continuous-wave (cw) laser light seeds the system and provides the wavelength tunability required by the experiment while overlapping its output with the dye's gain curve. The seeding light is

a) Electronic mail: Matthias.Verlinde@kuleuven.be aligned through the second part of the PDA setup, which comprises two or more amplifier dye cells, separated by optical elements to control the beam shape and minimize the Amplified Spontaneous Emission (ASE) contribution. The PDA system can also include a frequency conversion unit. The dye cells are pumped by a pulsed laser system defining the repetition rate and pulse length of the final output radiation. Due to its high output power, the most popular pump lasers used for this task are Q-switched Nd:YAG lasers, replacing older coppervapor lasers ${ }^{12}$. Owing to the high efficiency of the dye's gain medium, the combination of dye, a cw seed laser and a $10 \mathrm{kHz}$ pump laser can produce high-peak power and high-repetition rate laser pulses, with a Fourier-limited bandwidth.

Up until recently, however, the combination of high repetition rate, high pulse intensity and high resolution proved to be cumbersome. One of the main roadblocks is the amplitude modulation present in most commercial, high-repetition rate, Q-switched Nd:YAG lasers with a pulse length around $10 \mathrm{ns,} \mathrm{optimal} \mathrm{for} \mathrm{pumping} \mathrm{dye}$ lasers. These intensity modulations are caused by the beating of several longitudinal modes inside the laser cavity. The desired single-longitudinal-mode operation is difficult to achieve in the short-build up time and highgain operation of these Q-switched Nd:YAG lasers. In their laser cavity, the amount of round trips made by the oscillating photons is limited and as such any modeselective elements lack efficiency. Nd:YAG pump lasers with longer pulse lengths, typically employed for pumping solid-state Ti:Sa lasers, will, in comparison, intrinsi- 
cally limit the multi-mode operation. Because the PDA is a single pass amplifier, the dye's strong gain causes its population inversion to mimic the amplitude modulation present in the pump pulse. The exact conversion from the modulation frequency of the pump source to that of the dye depends on the lifetime of the dye's molecular transition pumped, as a slower response averages out the modulations. The single-pass nature of the amplifier results in a time modulation of the PDA output pulse, inducing undesirable sidebands to the frequency spectrum. Thus, a Nd:YAG pump laser operating in several longitudinal modes will necessarily cause a multi-mode behavior of the PDA laser system ${ }^{2}, 3,6,7,13,14$.

In the 1980's/1990's, when the PDA laser system was first applied, this problem was avoided by introducing new techniques to make the Nd:YAG lase in a single longitudinal mode. Several methods to achieve this were introduced: cw seeding, employing passive Q-switching, introducing extra mode-selecting elements, adding additional pulse delays, etc. ${ }^{2,13,15,16}$. The injection-seeding technique $^{17-20}$ proved to be the most popular. By introducing an additional $\mathrm{cw}$ master laser to seed the Nd:YAG cavity, single-longitudinal-mode operation could be achieved. This technique, however, was limited to the high-pulse intensity, low-repetition rate Nd:YAG lasers $(10-100 \mathrm{~Hz})$ that were available at that time, although, more recently, some work in the $1 \mathrm{kHz}$ repetition rate range can be found ${ }^{21}$. For the aforementioned applications, also high-repetition rate Nd:YAG lasers should be operated in a single longitudinal mode without excessively compromising its pulse intensity in order to remain efficient pump sources for dye lasers ${ }^{22}$.

In this article, we report the characterization of a new single-mode, $10 \mathrm{kHz}, \approx 5 \mathrm{~ns}$ pulse width $\mathrm{Nd}$ :YAG laser, operating at an average power of $40 \mathrm{~W}$, a pulse energy of $4 \mathrm{~mJ} /$ pulse and wavelength of $532 \mathrm{~nm}$, as well as its application in the In-Gas-jet Laser Ionization and Spectroscopy technique (IGLIS). The IGLIS technique is being developed to perform laser hyperfine spectroscopy of short-lived isotopes in a supersonic gas jet environment ${ }^{1,23-26}$. The cold, low-density gas jet provides ideal conditions for high-resolution spectroscopy on medium-heavy and heavy isotopes. To maximize the efficiency of laser ionization, to minimize the duty-cycle losses induced by the atoms of interest traveling at a longitudinal velocity of $550 \mathrm{~m} / \mathrm{s}$ in the jet, and to allow for high-resolution laser ionization spectroscopy without any spectral sidebands, transform-limited high-power laser pulses are required at $10 \mathrm{kHz}$ or higher repetition rate. To summarize, we report on the performance of a prototype single-longitudinal-mode pumped PDA, describing the reduction of the spectral sideband amplitude compared to the main resonance (refered to in the text as the reduction factor), as a direct comparison to demonstrate its suitability for in-gas-jet laser spectroscopy, by carrying out laser ionization spectroscopy studies in an Atomic Beam Unit (ABU). In Sec. II, the potential problems using a multi-longitudinal-mode (MLM) Nd:YAG laser for applications with the IGLIS technique are described. Sec. III presents a characterization of the new singlelongitudinal-mode (SLM) laser in the frequency and the time domain. Finally, a direct comparison of the SLMpumped PDA system's performance with that of a typical MLM-pumped system is described in Sec. IV.

\section{MULTI-LONGITUDINAL-MODE OPERATION}

To illustrate the problems that arise when performing narrowband laser spectroscopy with a PDA system pumped by a commercial MLM Nd:YAG laser, hyperfine spectroscopy was performed in an Atomic Beam Unit (ABU), see Fig. 1, on the $4 \mathrm{~s}^{2} \mathrm{~S}_{1 / 2}$ ground state (g.s.) to the $4 \mathrm{p}^{2} \mathrm{P}_{1 / 2}$ excited state (e.s.) transition in ${ }^{63,65} \mathrm{Cu}$. The ionization scheme is presented in Fig. 2. The laser light required to excite such a transition is obtained from the frequency-doubled output of the PDA laser system, pumped by a $90 \mathrm{~W}$ MLM Nd:YAG laser (INNOSLAB ISIII-E, Edgewave GmbH). The custom-made PDA system operated at the IGLIS laboratory (Sirah Lasertechnik $\mathrm{GmbH}$ ) is seeded by a diode laser (TA:pro, TOPTICA Photonics AG) at around $654.98 \mathrm{~nm}^{23}$. This diode laser offers a typical power of $150 \mathrm{~mW}$. The fundamental PDA output, lasing at the same wavelength as the $\mathrm{cw}$ diode, is guided to a nonlinear BBO crystal for frequency doubling, after which it is separated from the UV radiation by a set of Pellin-Broca prisms, see Fig. 3. The output power of the PDA is routinely found to be above $100 \mathrm{~mW}(10 \mu \mathrm{J}$ at $10 \mathrm{kHz})$. This is sufficient to saturate the transition in copper, which has a measured saturation intensity of $0.11(5) \mathrm{nJ} / \mathrm{mm}^{2}$. The ionization step at $287.98 \mathrm{~nm}$ is provided by a broadband $(\sim 2.5 \mathrm{GHz})$ tunable dye laser (CREDO, Sirah Lasertechnik GmbH) with also an integrated frequency conversion unit. This laser was pumped by a second $100 \mathrm{~W}$ MLM Nd:YAG laser (INNOSLAB ISIII-E, Edgewave GmbH). A power of $500 \mathrm{~mW}$ is routinely obtained for this wavelength. Both lasers were operated at a repetition rate of $10 \mathrm{kHz}$, synchronously triggered by a pulse generator (9530 Series Quantum Composers Inc.). The ABU provides a copper atom beam by a resistively heated graphite crucible. After laser ionization, these ions experience a two-stage acceleration region before a field-free drift towards a Multi Channel Plate (MCP) detector. At the MCP, the timeof-flight of the copper ions is recorded by a Time to Digital converter (TDC) to provide a mass selection with a mass resolving power $M / \delta M \approx 150$ for ${ }^{63,65} \mathrm{Cu}$. To map the $4 \mathrm{~s}^{2} \mathrm{~S}_{1 / 2}$ to $4 \mathrm{p}{ }^{2} \mathrm{P}_{1 / 2}$ hyperfine structure, the TA:pro's wavelength is scanned in the region 654.975$654.985 \mathrm{~nm}$ (in vacuum), controlled and stabilized via a wavelength meter (WS7-60, High Finesse GmbH) that is additionally supplied with a frequency stabilized HeNe laser (Model 32734, Research Electro-Optics, Inc.). The ion count rate is monitored as a function of the TA:pro's wavelength. A schematic overview of the setup used for these experiments can be found in Fig. 1. A more de- 
tailed discussion on the working principle of the IGLIS laboratory can be found in ${ }^{1,27-29}$. The results are shown in Fig. 2. A resonance linewidth of around $120 \mathrm{MHz}$ is achieved here, presenting a trade off between ionization efficiency and ionization laser power broadening ${ }^{30}$. Clearly, one can distinguish a whole array of additional resonances, not belonging to the well-known hyperfine structure of the copper transition probed. A description of the origin of these additional resonances and a detailed characterization will be done in the next section. A closer look reveals that each hyperfine resonance is copied at distinct frequency shifts from the expected value, $\Delta \nu_{s, n}=n \cdot \Delta \nu_{s}$, with an integer $n$ describing the harmonic order. Therefore, these additional resonances will be referred to as sidebands throughout the text. In this case, $\Delta \nu_{s}=800(2) \mathrm{MHz}$, which is derived by fitting the centroids of all distinguishable resonances. The amplitude of these sidebands, in the absence of laser saturation and with respect to the main hyperfine resonance, are constant in time and match well with the analytic description (in the absence of power broadening):

$$
R_{\Delta \nu_{s, n}}^{\mathrm{ion}}=\alpha \cdot\left(n \cdot \Delta \nu_{s}\right)+\beta,
$$

with $\alpha=-7(1) \cdot 10^{-6} \mathrm{MHz}^{-1}, \beta=0.10(1)$, and $n$ as high as 13. The reduction factor $R_{\Delta \nu_{s, n}}^{\text {ion }}$ is defined as follows: $R_{\Delta \nu_{s, n}}^{\text {ion }}=A_{\Delta \nu_{s, n}}^{\text {ion }} / A_{0}^{\text {ion }}$, with $A_{0}^{\text {ion }}$ and $A_{\Delta \nu_{s, n}}^{\text {ion }}$ the ion count rate obtained at true resonance and at a sideband frequency shift $\Delta \nu_{s, n}$, respectively. In case the excitation laser intensity is increased up to or beyond the saturation intensity, $R_{\Delta \nu_{s, n}}^{\text {ion }}$ will increase drastically.

As the primary objective of the IGLIS technique is to perform laser ionization spectroscopy on heavy elements, that are often only produced in minute amounts resulting in spectra with limited statistics, at an expected resolution between $150 \mathrm{MHz}$ and $200 \mathrm{MHz}$, the presence of these sidebands will prevent a conclusive analysis. The situation illustrated here, represents already an improvement of the standard operation of the PDA as the relative arrival of the pump laser pulses at the dye cells in the PDA were delayed, as in ${ }^{13,31}$, explaining the absence of the $\Delta \nu_{s, n}$ resonances for odd $n$ in Fig. 2 (compare dotted and solid lines in Fig. 2). However, it was deemed practically impossible to suppress all sideband orders adequately for operation of such a laser system in the IGLIS technique.

\section{SINGLE-LONGITUDINAL-MODE LASER}

To rectify the issues brought forward in Sec. II, a new prototype single-longitudinal-mode (SLM) Nd:YAG laser, developed by Edgewave $\mathrm{GmbH}$, was introduced to the setup, Fig. 1. This $532 \mathrm{~nm}, 40 \mathrm{~W}$ and $10 \mathrm{kHz}$ laser is used to pump the PDA system. Due to the lower laser power and mainly the non-optimal beam shape of the present prototype model, the output power of the PDA system is limited to $10 \mathrm{~mW}$ at $10 \mathrm{kHz}$ and a wavelength of $327 \mathrm{~nm}$. However, this power is still more

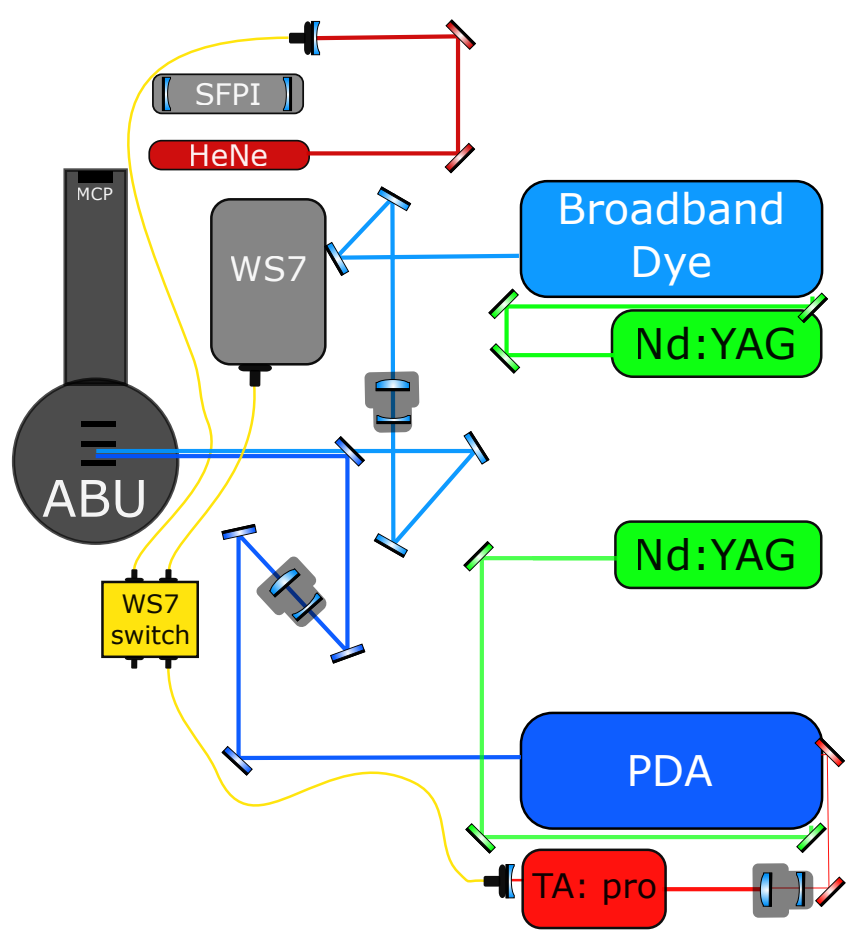

FIG. 1. Schematic overview of the setup used to determine the hyperfine parameters of the $4 \mathrm{~s}^{2} \mathrm{~S}_{1 / 2}$ to $4 \mathrm{p}{ }^{2} \mathrm{P}_{1 / 2}$ transition (see text for details). The Nd:YAG laser pumping the PDA is either the commercial INNOSLAB ISIII-E, see Sec. II, or the prototype SLM, see Sec. IV.

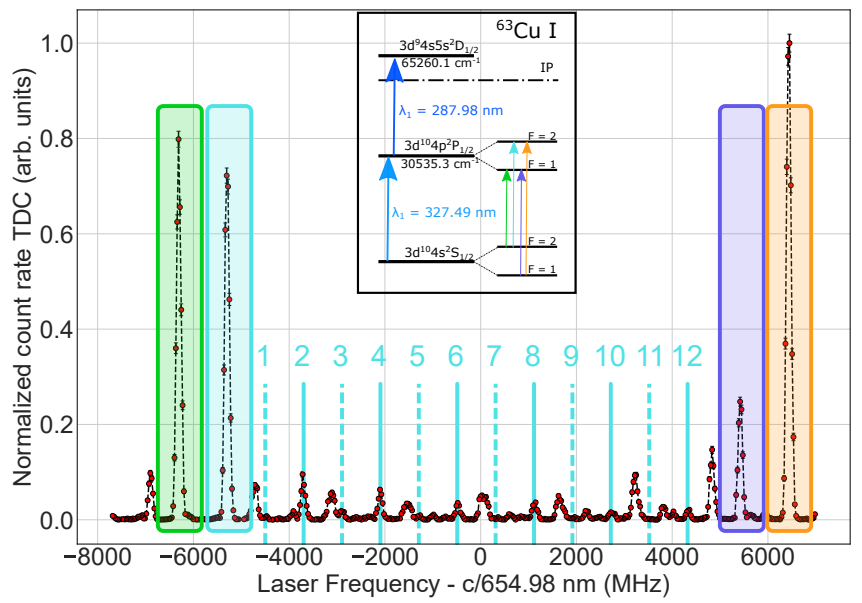

FIG. 2. Hyperfine spectrum of the $4 \mathrm{~s}^{2} \mathrm{~S}_{1 / 2}$ to $4 \mathrm{p}^{2} \mathrm{P}_{1 / 2}$ transition in ${ }^{63} \mathrm{Cu}$. The true transitions are highlighted with the color matching that on the ionization scheme. All other resonances are sidebands related to one of the four true hyperfine transitions. For clarity, the $\mathrm{F}=2$ to $\mathrm{F}=2$ transition (light blue color) is used as a reference to show this sequence of sidebands. These sidebands show no overlap, within the experimental precision, with any other sidebands, hence the choice of this transition. The odd numbers are represented by the dotted lines, while those solid represent the even ones. 


\begin{tabular}{c|c}
\hline \hline & SLM Nd:YAG \\
\hline Repetition Rate & $10 \mathrm{kHz}$ \\
\hline Wavelength & $532 \mathrm{~nm}$ \\
\hline Average Power & $40 \mathrm{~W}$ \\
\hline Pulse Energy & $4 \mathrm{~mJ}$ \\
\hline Pulse Length & $\approx 5 \mathrm{~ns}$ \\
\hline Peak Power & $0.9 \mathrm{MW}$ \\
\hline Beam Quality & $\mathrm{M}^{2}<1.3$ \\
\hline Beam Diameter & $3 \mathrm{~mm}$ \\
\hline Pulse Energy Stability & $2 \% \mathrm{rms}$ \\
\hline Pulse Timing Stability & $3 \mathrm{~ns}$ \\
\hline \hline
\end{tabular}

TABLE I. Specifications of the prototype SLM Nd:YAG laser

than adequate to saturate most strong transitions in laser spectroscopy. The dye solution used for the PDA cells consists of DCM with a concentration of $0.450 \mathrm{~g} / 1$ and $0.300 \mathrm{~g} / \mathrm{l}$ in the preamplifier and main amplifier, respectively, in a mixture of $25 \%$ ethanol and $75 \%$ dimethyl sulfoxide (DMSO). The SLM Nd:YAG pump laser comprises a diode pumped, EO Q-switched laser equipped with an actively-stabilized intracavity etalon arrangement seeding an Innoslab amplifier with frequency doubling unit ${ }^{32}$. The active stabilization procedure is based on the pulse area stability, which is enhanced in single mode operation to $\approx 0.2 \%-0.5 \%$. The two parameters available to stabilize the etalon are the cooling water temperature and total current sent to the diode pumping array, which allow the laser to work in a single longitudinal mode in a narrow temperature range. This set current defines the final pulse width (within the range $4-6 \mathrm{~ns}$ ) and timing of the output laser pulse. Once outside this optimal range, the laser was working in MLM operation. A schematic layout of the SLM laser is shown in Fig. 3 and its main characteristics are summarized in Tab. I.

To provide a full description of the mode structure of the Nd:YAG laser and the PDA output itself, the prototype laser was operated in two different ways, singlelongitudinal mode (SLM) or unstable multi-longitudinal mode (MLM). The MLM operation, indicating a improper stabilization of the intracavity etalon, can be compared to the original MLM Nd:YAG laser, see Sec. II, although with a decreased number of sideband modes due to the presence of the etalon inside the cavity. The time profiles and frequency components of the laser pulses were investigated by a fast photodiode and Scanning Fabry Pérot Interferometer (SFPI), respectively.

\section{A. Time Profile}

In MLM operation, i.e. with a non-optimized set of parameters in the Nd:YAG pump laser, the intensity time profiles and corresponding live Fast-Fourier Transformations (FFT) of the pulses from the Nd:YAG laser, PDA

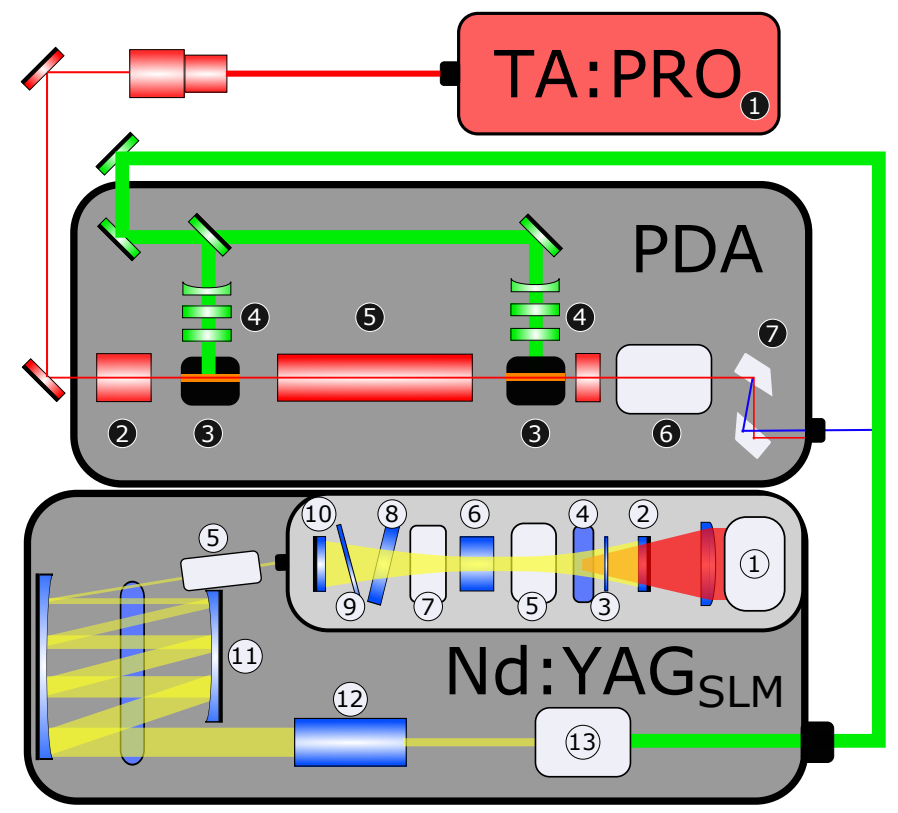

FIG. 3. Schematic overview of the PDA laser system (white numbering) and SLM Nd:YAG pump laser (black numbering). The single mode cw diode laser (1) seeds the PDA system. After passing through beam shaping optics (2), the seed beam arrives at the preamplifier dye cell (3), which is pumped by the SLM laser through beam shaping optical elements (4). The amplified beam passes several more beam and single pass optics (5) before arriving at the second, main amplifier dye cell. The final amplified fundamental beam is focused into a BBO nonlinear crystal (6) with corresponding Pellin-Broca optical setup (7). For the SLM Nd:YAG laser, the seed laser consists of a diode pump laser (1) that is focused into the Nd:YAG crystal (4) through the end mirror (2). The rest of the cavity houses a 45 degree quarter wave plate and a Faraday isolator (5), to avoid spatial hole burning, a polarizer (6), an Electro Optical Modulator (EOM, 7), a $5 \mathrm{~mm}(8)$ and $1 \mathrm{~mm}$ (9) dual etalon setup and the output mirror (10). Next, The light of the seed laser is sent through an INNOSLAB amplifier (11), pumped by a diode stack (not shown here). After amplification, the fundamental laser beam of $1064 \mathrm{~nm}$ is guided through beam shaping optics (12) to obtain an optimal beam profile for frequency doubling in a nonlinear crystal (13). The components of the SLM laser are not drawn to scale nor completeness, they serve indicative purposes only.

fundamental output and PDA frequency doubled output were recorded, see Fig. 4. These time profiles were measured by sending a pickoff of the laser light to a fast InGaAs photodiode (ET-3010, Electro-Optics Technology, bandwidth $>2 \mathrm{GHz}$ ), read out by an oscilloscope (RTO 1044, Rhode\&Schwarz, bandwidth 4 GHz). Owing to longitudinal mode competition in the Nd:YAG cavity, which is a common feature for these kind of lasers, mode beating at distinct frequencies, $\Delta \nu_{s, n}=n \cdot \Delta \nu_{s}$, with $\Delta \nu_{s}$ equal to the Free Spectral Range (FSR) of the laser cavity, is observed in the intensity profiles of the laser output, resulting in a modulated Gaussian time profile ${ }^{33}$. For this specific resonator, an FSR of $681(2) \mathrm{MHz}$ was 
determined from the Fourier transformation of the time profiles. Because of the fast response of the molecular transitions involved in the dye gain medium, this modulated Gaussian time profile is transferred from the pump laser to the time-dependent population inversion created in the dye medium. The high gain of the dye medium causes the laser light of the PDA fundamental and frequency doubled output to exhibit similar modulations in their intensity time profile with an amplitude depending on the dye's lifetime. These amplitude modulations induce unwanted spectral components causing the observed sidebands in spectroscopy.

Optimizing the pump laser parameters to work in SLM, results in the data shown in Fig. 5. Although exhibiting small traces of a beat frequency at the same frequency $\Delta \nu_{s}$, the intensity profile hints towards a strongly improved SLM operation of the Nd:YAG pump laser in a Gaussian pulse length of 5.65(5) ns. The exact pulse length of the laser is strongly dependent on its current settings, which are capable of stabilizing the etalon, as mentioned earlier.

The laser intensity, centered around $t=0$, as a function of time and their corresponding FFT's can be described, in a first approximation, as follows:

$$
\begin{aligned}
& I(t)_{\lambda}=\frac{A}{\sigma \sqrt{2 \pi}} e^{-t^{2} / 2 \sigma^{2}} \times \\
& \left(1+\sum_{n=1}^{n_{f}} A_{n}^{\lambda} \cos \left(2 \pi \Delta \nu_{s, n} t\right)\right), \\
& \mathcal{F}\left\{I(t)_{\lambda}\right\}(\omega)=A e^{-\omega^{2} \sigma^{2} / 2}+ \\
& \sum_{n=1}^{n_{f}} \frac{A A_{n}^{\lambda}}{2} e^{-\left(\omega \pm 2 \pi \Delta \nu_{s, n}\right)^{2} \sigma^{2} / 2}
\end{aligned}
$$

with $\sigma$ representing the pulse width, $n_{f}$ the total amount of available modulation frequencies, $\lambda=532,654 \mathrm{~nm}$ and the factor $A_{n}^{\lambda}$, also referred to as modulation depth. The focus in the following will be on $A_{1}^{\lambda}$, as it most often represents the highest intensity sideband. The frequency doubled output of the PDA is constructed from $\left|I(t)_{\lambda}\right|^{2}$. Taking the ratio of the peak centered at zero frequency and the first sideband in the FFT spectrum, reveals thus the modulation intensity $A_{1}^{\lambda}$. Focusing on the $532 \mathrm{~nm}$ light, as this will be easiest to monitor during experiments, it was observed that the beat mode intensity $A_{1}^{532}$ could be reduced from values $>35 \%$ for MLM operation to at least $A_{1}^{532, \mathrm{ftt}}(\mathrm{SLM}) \approx 1.5 \%$ in stable SLM operation. Information on $A_{1}^{654,327}$ and, thus, the transformation of $A_{1}^{532}$ to $A_{1}^{654,327}$ is also available through a pulse-by-pulse analysis using two fast InGaAs photodiodes.

From laser ionization spectroscopy in the ABU, see Sec. II,IV, the reduction factor $R_{\Delta \nu_{s, 1}}^{\text {ion }}=$ $A_{\Delta \nu_{s, 1}}^{\text {ion }}(\mathrm{MLM}) / A_{0}^{\text {ion }}(\mathrm{MLM})$, or relative strength of the sideband resonance in comparison to the main one, is
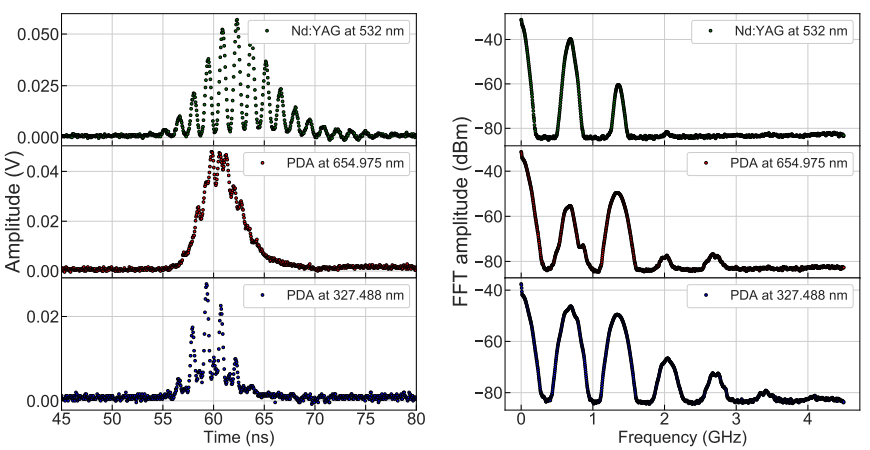

FIG. 4. Time profiles of the Nd:YAG laser operating at $532 \mathrm{~nm}$ in MLM operation, the PDA at $654.975 \mathrm{~nm}$ and the PDA at the doubled frequency output. The time profiles are accompanied by their Fast Fourier Transform (FFT), measured by the oscilloscope directly. The FFT amplitude represents an average over 1000 traces, while all different profiles were measured subsequently. This means that the exact timing of the pulses shown is arbitrary. Also, the pulse length of individual pulses can not be compared directly as different settings were used to keep the laser operating in MLM. The pump laser was lasing in different longitudinal modes simultaneously, revealing distinct beat frequencies.

determined to be $\approx 5-10 \%$, comparable to previous results discussed in Sec. II. To determine the transformation that translates the modulation intensity $A_{1}^{532}$, obtained via a live FFT capture of the Nd:YAG pump laser, see Eq. 2, to the amplitude of the resonances, $A_{\Delta \nu_{s, 1}}^{\text {ion }}$, a laser-atom model is used based on the Von Neumann equations ${ }^{30}$. From the measured pulse shape and other experimental data such as the characteristics of the laser pulses, the atomic levels involved, etc. the model produces the occupation of each atomic level as a function of time and frequency. Introducing the modulation to the excitation laser results in the occurrence of spectral sidebands in the simulated ionization spectra. With the measured time profiles as a starting point, the model predicts $R_{\Delta \nu_{s, 1}}^{\text {ion }}=A_{\Delta \nu_{s, 1}}^{\text {ion }}(\mathrm{MLM}) / A_{0}^{\text {ion }}(\mathrm{MLM})>7.5 \%$ for $A_{1}^{532}>35 \%$ while for SLM operation $R_{\Delta \nu_{s, 1}}^{\text {ion }}=$ $A_{\Delta \nu_{s, 1}}^{\text {ion }}(\mathrm{SLM}) / A_{0}^{\text {ion }}(\mathrm{SLM})<0.015 \%$ for $A_{1}^{532}<1.5 \%$. A 25-fold reduction in the modulation intensity of the Nd:YAG laser light produces, thus, a 500-fold reduction in $R_{\Delta \nu_{s, 1}}^{\text {ion }}$. These simulations were performed assuming an excitation laser intensity at least one order of magnitude below the saturation value. Increasing the laser power close to or beyond the saturation value will have a severe impact on these results, increasing the amplitude of the sideband resonances significantly.

\section{B. Frequency Profile}

The frequency components of both the Nd:YAG pump laser and the fundamental output of the PDA laser were analyzed by an Scanning Fabry-Pérot Interferome- 

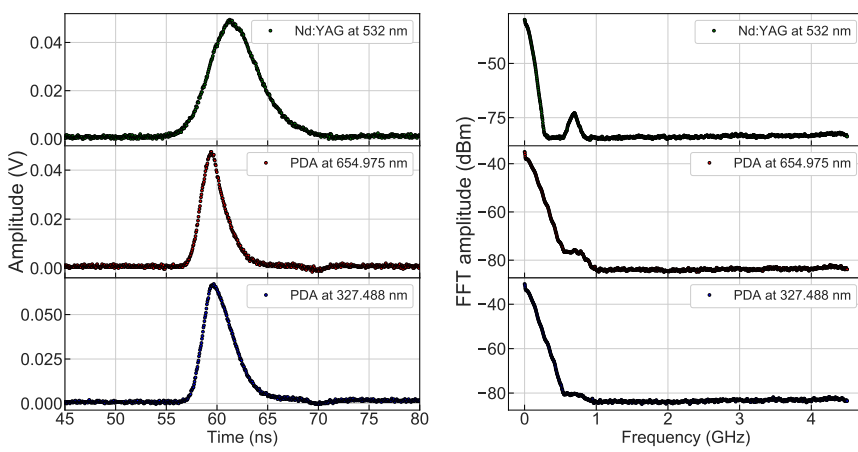

FIG. 5. Time profiles of the Nd:YAG laser operating at $532 \mathrm{~nm}$ in SLM, the PDA at $654.975 \mathrm{~nm}$ and the PDA at the doubled output. The same comments as Fig. 4 apply also here. The pump laser was lasing in one single longitudinal mode, almost reducing the beat frequency component to noise level.

ter (FPI-100-0500-1, Toptica Photonics AG), with a Free Spectral Range (FSR) of $4 \mathrm{GHz}$. The results of a single voltage sweep of the piezo inside the SFPI cavity are shown in Fig. 6, for MLM and SLM operations of the pump laser. The $10 \mathrm{kHz}$ pulsed light is accompanied, simultaneously, by a continuous-wave, frequencystabilized HeNe laser (Model 32734, Research ElectroOptics, Inc.) that is used as a frequency calibration of the different laser fringe timings. The analysis for this data is similar to that presented $\mathrm{in}^{29}$. For the $532 \mathrm{~nm}$ light, in MLM operation, clearly three modes are competing with each other inside the laser cavity, causing strong pulse-to-pulse mode fluctuations in the observed signal. The difference between two neighboring modes is found to be $\Delta \nu_{s}=679(9) \mathrm{MHz}$, obtained after using the HeNe for a time-frequency conversion, which agrees with the beat frequency observed in the time profiles. Because mainly three modes compete in the pump laser, two beat frequencies will be most significant, see Fig. 4 . The modulated Gaussian time profiles is also observed in Fig. 4 for the PDA output. The SFPI analysis of this light reveals, in agreement with a Fourier transform of the time profile, that this is translated into singlelongitudinal-mode laser radiation at $654.98 \mathrm{~nm}$ (the specific SFPI used here is not sensitive to the UV light) accompanied by two sideband modes that are shifted from the main frequency component by $\Delta \nu_{s}$ and $2 \Delta \nu_{s}$, which are induced by the three competing modes in the pump laser light, see Fig. 6 (a) and (b). From this dataset one finds that $\Delta \nu_{s}=681(2) \mathrm{MHz}$. Also here, the mode intensities fluctuate in a pulse-to-pulse manner. The sideband frequencies exhibit amplitudes $R_{\Delta \nu_{s, 1}}^{\mathrm{sfpi}}=4(1) \%$ of the main frequency component. Operating the Nd:YAG laser in SLM results in the SFPI spectrum of Fig. 6 (c). Clearly, only one mode persists, with a stable pulse-topulse amplitude which is strongly different from that in MLM operation. As seen in Fig. 5, almost no modulated time profile is observed in this mode of operation.
This is translated to the results of an SFPI sweep of the PDA fundamental output, Fig. 6 (d). True single-mode operation of the PDA laser is achieved within the sensitivity of the SFPI device, $1: 200$. The bandwidth of the PDA laser, pumped by the single mode Nd:YAG laser is measured to be 97.6(4) MHz. In the Fourier limit, this corresponds to a Gaussian shaped laser pulse with 4.43(3) ns, corresponding to the measured pulse width at the specific settings of the laser at that time.

A final test was performed with a home-made FPI device, with a FSR of 11.8(2) GHz. The $532 \mathrm{~nm}$ light from the $\mathrm{Nd}$ :YAG laser is overlapped with the frequency stabilized HeNe laser at the entrance of the FPI and read out interchangeably. After passing a lens arrangement, the light is collected on a CCD camera beam profiler (BC106NVIS/M, Thorlabs Inc.) Clearly three modes are oscillating in the laser cavity, confirming previous measurements. The frequency difference $\Delta \nu_{s}=670(10)$ between the modes is in agreement with the other techniques.

\section{LASER-SPECTROSCOPY RESULTS}

To showcase the performance of the SLM pumped PDA, and its applicability for future research with the IGLIS technique, the same laser ionization spectroscopy experiment as presented in Sec. II was performed. The sole difference being the prototype SLM Nd:YAG laser pumping the PDA system. A hyperfine spectrum obtained through a frequency scan of the TA:pro diode laser, seeding the PDA laser system, is shown in Figs. 7 and 8 for ${ }^{63} \mathrm{Cu}$. For MLM operation of the Nd:YAG laser, pumping the PDA, the spectrum exhibits not only the true hyperfine components of the $4 \mathrm{~s}^{2} \mathrm{~S}_{1 / 2}$ to $4 \mathrm{p}^{2} \mathrm{P}_{1 / 2}$ transition, but also additional resonances, confirming the results of Sec. II. These sidebands are related, one-byone, to a single hyperfine component shifted by $n \cdot \Delta \nu_{s}$ in the frequency domain. Similar results have been obtained for ${ }^{65} \mathrm{Cu} . \Delta \nu_{s}$ can be determined from these measurements to be $\Delta \nu_{s}=681(4) \mathrm{MHz}$. The sidebands are, thus, in agreement with those observed in the SFPI and time-domain sweeps of Figs. 6 and 5. As expected, the three main oscillating modes in the Nd:YAG laser cavity cause two sidebands to appear in the spectroscopy results. The amplitude ratio of the sidebands with respect to the true hyperfine component corresponds to $R_{\Delta \nu_{s, n}}^{\text {ion }} \approx 5-10 \%$, depending on the measurement. These ratios are comparable or higher than those obtained with the SFPI measurements, influenced by the saturation effects of the laser ionization process. As discussed earlier, the presence of such sidebands in the hyperfine spectra of unmeasured isotopes/isomers, will either skew the experimental results significantly, or at least severely hamper the analysis, as exemplified here by the spectroscopy measurements on the $4 \mathrm{~s}^{2} \mathrm{~S}_{1 / 2}$ ground state (g.s.) to the $4 \mathrm{p}^{2} \mathrm{P}_{1 / 2}$ excited state (e.s.) transition in ${ }^{63} \mathrm{Cu}$.

For the Nd:YAG laser in single-mode operation, a flat baseline, up to a sensitivity of $1: 200$, is obtained, only 

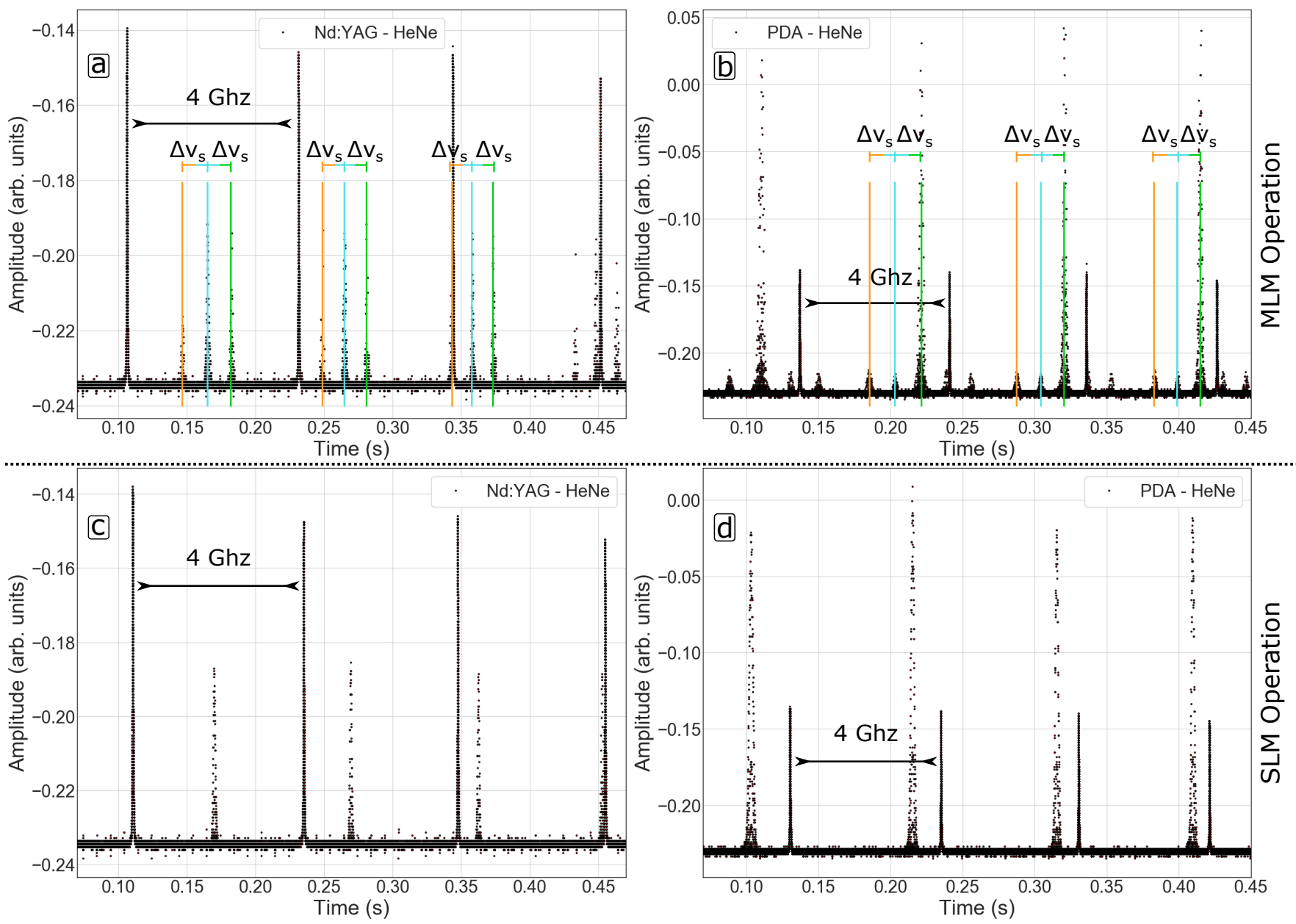

FIG. 6. SFPI traces of both the Nd:YAG $532 \mathrm{~nm}$ and the PDA $654.975 \mathrm{~nm}$ light operating at $10 \mathrm{kHz}$ repetition rate in different operating modes of the Nd:YAG laser. A frequency stabilized HeNe is included for time to frequency calibration. In (a), the Nd:YAG laser light shows three oscillating modes that are transferred to the PDA output as sideband frequencies alongside the main frequency band determined by the $\mathrm{cw}$ diode laser seeding the PDA system, visible in (b). The symmetric occurrence of the sideband modes in (b) is due to the \pm term in Eq. 2. In (c) and (d), when the laser is run in single-mode operation, both the Nd:YAG laser light and the PDA output show a single oscillating mode within the sensitivity limits of the SFPI device.

disturbed by the true resonances of the $4 \mathrm{~s}^{2} \mathrm{~S}_{1 / 2}$ to $4 \mathrm{p}$ ${ }^{2} \mathrm{P}_{1 / 2}$ hyperfine structure. Analysis of these results, via the SATLAS python package, delivers the hyperfine parameters, $A\left({ }^{63} \mathrm{Cu}\right.$,g.s. $)=5866.2(7) \mathrm{MHz}, A\left({ }^{63} \mathrm{Cu}\right.$,e.s. $)=$ $505.2(7) \mathrm{MHz}, A\left({ }^{65} \mathrm{Cu}\right.$,g.s. $)=6285.5(12) \mathrm{MHz}$ and $A\left({ }^{65} \mathrm{Cu}\right.$,e.s. $)=540.0(11) \mathrm{MHz}$, in agreement with lit-

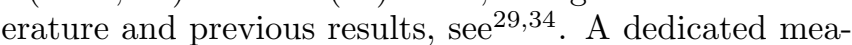
surement was done to obtain the ratio between the true ${ }^{63,65} \mathrm{Cu}$ resonances and closest sidebands. After confirming that laser power saturation was not present, the accumulated number of counts at a true resonance and its closest, undisturbed, sideband were monitored. While the simulations using the measured time profiles indicate a $R_{\Delta \nu_{s, 1}}^{\mathrm{ion}}<0.015 \%$, the spectroscopy measurements show that $R_{\Delta \nu_{s}}^{\text {ion }} \leq 0.2 \%$. Because the spectral tails of the closest true ${ }^{63} \mathrm{Cu}$ hyperfine resonance, at the minimal broadening possible in this $\mathrm{ABU}$, have a similar reduction factor with respect to the peak amplitude, closing in on the expected $R_{\Delta \nu_{s, 1}}$ remains impossible in this way. Laser spectroscopy in a supersonic gas jet is, currently, not only used as a laser spectroscopy tool to obtain hyperfine parameters, it is also employed at KU Leuven as a tool to characterize the performance of high-Mach number de Laval nozzles by using the $4 \mathrm{~s}^{2} \mathrm{~S}_{1 / 2}$ to $4 \mathrm{p}$ ${ }^{2} \mathrm{P}_{1 / 2}$ transition in ${ }^{63,65} \mathrm{Cu}^{26}$. The quality of these nozzles is essential to provide the coldest, lowest density gas jets, maximizing the final measurement resolution of the IGLIS technique while maintaining a relatively homogeneous flow profile for at least $55 \mathrm{~mm}$. Also here, the availability of a single-mode pump laser providing quasi transform-limited laser pulses is a leap forward. As the precision on the final Mach number observable is determined by the defining properties of a spectroscopic Voigt fit on the hyperfine resonances obtained either via the Planer Laser Induced Fluorescence Spectroscopy (PLIFS) method, as $\mathrm{in}^{26}$, or resonant ionization 
spectroscopy, any present spectral sidebands will require more stringent assumptions on the fit parameters and, thus, increase the systematic uncertainty.

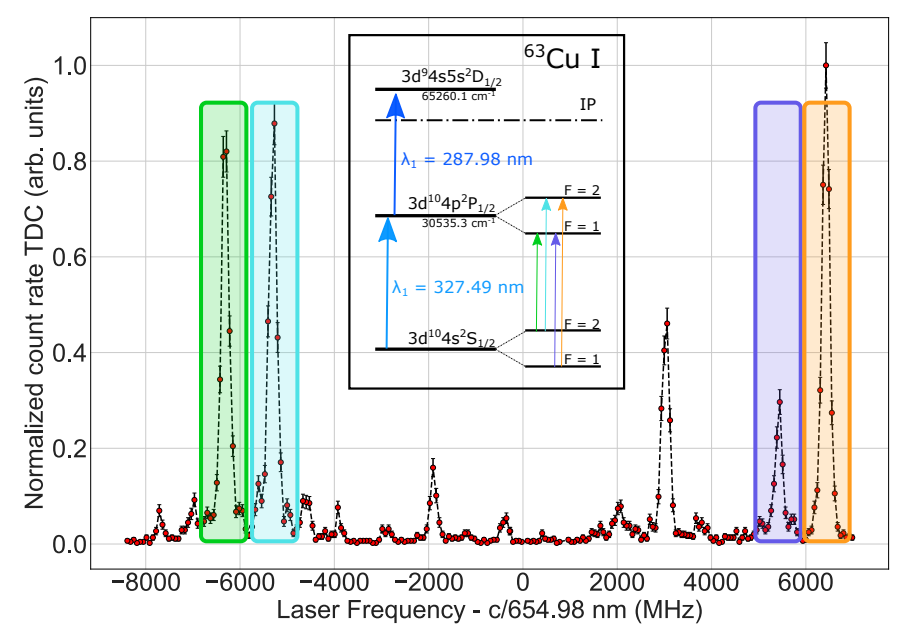

FIG. 7. Obtained hyperfine spectrum of the $4 \mathrm{~s}^{2} \mathrm{~S}_{1 / 2}$ to $4 \mathrm{p}^{2} \mathrm{P}_{1 / 2}$ transition in ${ }^{63} \mathrm{Cu}$ for the pump laser operating in MLM. A multitude of sidebands appear alongside the true hyperfine resonances, hindering a clear analysis of the nuclear parameters and potentially skewing the results. The ionization scheme is shown with the color code indicating the different transitions. The wavelength is quoted in vacuum.

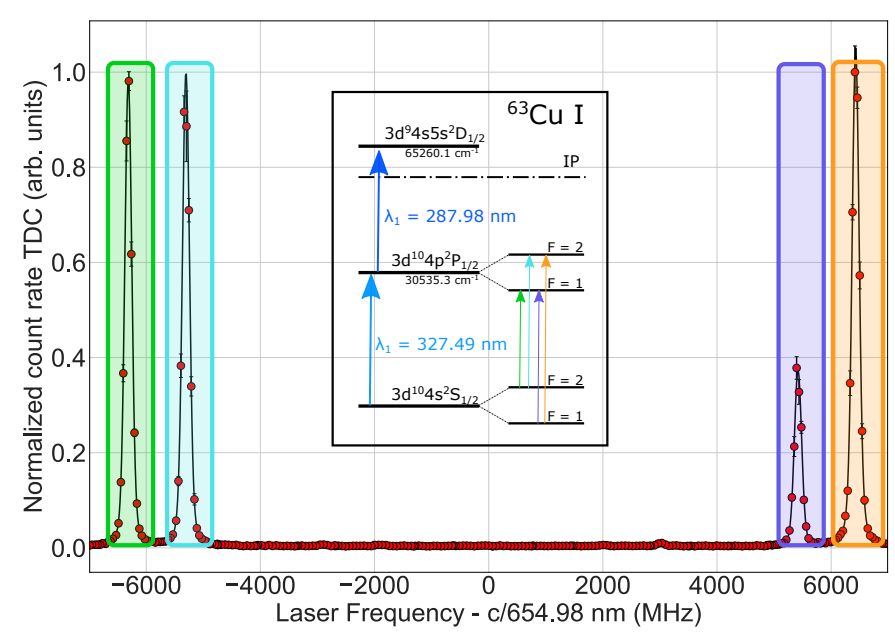

FIG. 8. Obtained hyperfine spectrum of the $4 \mathrm{~s}^{2} \mathrm{~S}_{1 / 2}$ to $4 \mathrm{p}$ ${ }^{2} \mathrm{P}_{1 / 2}$ transition in ${ }^{63} \mathrm{Cu}$ for the pump laser operating in a single axial mode. Only the true hyperfine resonances appear from the background, greatly facilitating the analysis. At a sensitivity level of $1: 200$, fake resonances appear at a frequency shift of $3.5 \mathrm{GHz}$ from their respective hyperfine transitions. These resonances originate from the TA:pro diode laser and presents the subsequent limiting factor. A fit to the hyperfine spectrum is provided in addition to the data. The ionization scheme is shown with the color code indicating the different transitions. The wavelength is quoted in vacuum.

\section{SUMMARY}

In this article, a PDA laser system is shown to be capable of producing high-power, high-repetition rate, near Fourier-limited laser pulses of a few nanoseconds pulse length. The PDA is pumped by a newly-developed prototype $10 \mathrm{kHz} \mathrm{Nd}$ :YAG laser whose single-longitudinal mode operation minimizes any amplitude modulations in the time profile of the Nd:YAG laser pulse. As any temporal modulation induces undesirable frequency components in the spectrum of the PDA laser, which are detrimental for high-resolution laser spectroscopy studies, this development was essential to optimize the operation of the $10 \mathrm{kHz} \mathrm{Nd}$ :YAG pumped PDA system and provide Fourier-limited behavior. The capability of the full laser system running either in MLM or SLM operation was compared by studying both the time behavior, via a large-bandwidth scope, and the frequency behavior, using an SFPI and laser ionization spectroscopy. Both methods show a reduction of the beating modes by a at least one order of magnitude. This lower limit was due to the specific sensitivities of the applied methods. For the final purpose of this system, performing highresolution laser spectroscopy in a gas-jet environment, results from laser ionization spectroscopy and the time behavior translate in a reduction of the resonant sideband amplitudes from $\approx 5-10 \%$ to al least $<0.2 \%$ of the true hyperfine resonance amplitudes, when comparing MLM to SLM operation of the Nd:YAG pumped PDA system. Simulations with a benchmarked model predict another order magnitude further reduction. After demonstrating the capabilities of the prototype SLM pump laser, a new version (PXn300-2-GF-SLM, Edgewave GmbH) was developed by Edgewave. Besides improvements to the SLM operational stability, the pulse length and output power are increased to $\approx 8 \mathrm{~ns}$ and $65 \mathrm{~W}$ at $15 \mathrm{kHz}$, respectively. In addition, the reduction factor is further increased with respect to the prototype laser. Finally, also the SLM stabilization procedure was strongly improved with the optimal cavity temperature range now solely controllable by the laser chiller's output temperature, which does not influence the laser pulse characteristics. This Nd:YAG laser will be used for the PDA system in future applications of the IGLIS technique at KU Leuven.

\section{ACKNOWLEDGMENTS}

The authors would like to thank Keming $\mathrm{Du}$ from Edgewave $\mathrm{GmbH}$ for the fruitful collaboration and the development of the Nd:YAG laser prototype and B. Marsh for providing the fast photodiode. This work has received funding from Research Foundation Flanders (FWO, Belgium), Excellence of Science program (EOS, FWO-FNRS, Belgium), by GOA/2015/010 (BOF $\mathrm{KU}$ Leuven), the Interuniversity Attraction Poles Programme initiated by the Belgian Science Policy Office (BriX network P7/12), from the European Union's 
Horizon 2020 research and innovation programme under grant agreement No 654002 (ENSAR) and from the European Research Council under the European Union's Seventh Framework Programme ERC-2011-AdG-291561HELIOS. M.V. is supported by an FWO grant (Aspirant $-1121820 \mathrm{~N})$.

\section{DATA AVAILABILITY STATEMENT}

The data that support the findings of this study are available from the corresponding author upon reasonable request.

${ }^{1}$ S. Raeder, B. Bastin, M. Block, P. Creemers, P. Delahaye, R. Ferrer, X. Fléchard, S. Franchoo, L. Ghys, L. P. Gaffney, C. Granados, R. Heinke, L. Hijazi, M. Huyse, T. Kron, Y. Kudryavtsev, M. Laatiaoui, N. Lecesne, F. Luton, I. D. Moore, Y. Martinez, E. Mogilevskiy, P. Naubereit, J. Piot, S. Rothe, H. Savajols, S. Sels, V. Sonnenschein, E. Traykov, C. Van Beveren, P. Van Den Bergh, P. Van Duppen, K. Wendt, and A. Zadvornaya, Nuclear Instruments and Methods in Physics Research, Section B: Beam Interactions with Materials and Atoms 376, 382 (2016).

2 J. D. Corless, J. A. West, J. Bromage, and C. R. Stroud, Review of Scientific Instruments 68, 2259 (1997).

${ }^{3}$ J. F. Black and J. J. Valentini, Applied Optics 33, 3861 (1994).

${ }^{4}$ G. Eesley, M. Levenson, D. Nitz, and A. Smith, IEE Journal of Quantum Electronics 16, 113 (1980).

${ }^{5}$ A. Farkas and J. Eden, IEE Journal of Quantum Electronics 29, 2923 (1993).

${ }^{6}$ E. S. Lee and J. W. Hahn, Optics Letters 21, 1836 (1996)

${ }^{7}$ P. Drell and S. Chu, Optics Communications 28, 343 (1979).

${ }^{8}$ E. Cromwell, T. Trickl, Y. T. Lee, and A. H. Kung, Review of Scientific Instruments 60, 2888 (1989).

${ }^{9}$ X. Wang, T. Kobayashi, Y. Matsuo, and T. Nakajima, Optics Communications 324, 168 (2014).

${ }^{10}$ S. Lavi, G. Bialolanker, M. Amit, D. Belker, G. Erez, and E. Miron, Optics Communications 60, 309 (1986).

${ }^{11}$ S. Raeder, R. Ferrer, C. Granados, M. Huyse, T. Kron, Y. Kudryavtsev, N. Lecesne, J. Piot, J. Romans, H. Savajols, P. Van Duppen, and K. D. Wendt, Nuclear Instruments and Methods in Physics Research, Section B: Beam Interactions with Materials and Atoms 463, 86 (2020).

${ }^{12}$ C. Schulz, E. Arnold, W. Borchers, W. Neu, R. Neugart, M. Neuroth, E. W. Otten, M. Scherf, K. Wendt, P. Lievens, Y. A. Kudryavtsev, V. S. Letokhov, V. I. Mishin, and V. V. Petrunin, Journal of Physics B: Atomic, Molecular and Optical Physics 24, 4831 (1991).

${ }^{13}$ P. Kumar and R. S. Bondurant, Applied Optics 22, 1284 (1983).

${ }^{14}$ Z. W. Li, C. Radzewicz, and M. G. Raymer, Optics Letters 12, 416 (1987).

${ }^{15}$ Y. Isyanova and D. Welford, Optics Letters 24, 1035 (1999).

${ }^{16}$ D. Voss and L. Goldberg, IEE Journal of Quantum Electronics 21, 106 (1985).

${ }^{17}$ Y. K. Park, G. Giuliani, and R. L. Byer, Optics Letters 5, 96 (1980).
${ }^{18}$ Y. K. Park, G. Giuliani, and R. L. Byer, IEEE Journal of Quantum Electronics 20, 117 (1984).

${ }^{19}$ R. L. Schmitt and L. A. Rahn, Applied Optics 25, 629 (1985).

${ }^{20}$ R. E. Teets, IEE Journal of Quantum Electronics 20, 326 (1984).

${ }^{21}$ J. Wang, R. Zhu, J. Zhou, H. Zang, X. Zhu, and W. Chen, Chinese Optics Letters 9, 081405 (2011).

${ }^{22}$ I. Freitag, A. Tünnermann, and H. Welling, Optics Letters 22, 706 (1997).

${ }^{23}$ Y. Kudryavtsev, P. Creemers, R. Ferrer, C. Granados, L. P. Gaffney, M. Huyse, E. Mogilevskiy, S. Raeder, S. Sels, P. Van Den Bergh, P. Van Duppen, and A. Zadvornaya, Nuclear Instruments and Methods in Physics Research, Section B: Beam Interactions with Materials and Atoms 376, 345 (2016).

${ }^{24}$ Y. Kudryavtsev, R. Ferrer, M. Huyse, P. Van den Bergh, and P. Van Duppen, Nuclear Instruments and Methods in Physics Research Section B: Beam Interactions with Materials and Atoms 297, 7 (2013).

${ }^{25}$ R. Ferrer, A. Barzakh, B. Bastin, R. Beerwerth, M. Block, P. Creemers, H. Grawe, R. de Groote, P. Delahaye, X. Fléchard, S. Franchoo, S. Fritzsche, L. P. Gaffney, L. Ghys, W. Gins, C. Granados, R. Heinke, L. Hijazi, M. Huyse, T. Kron, Y. Kudryavtsev, M. Laatiaoui, N. Lecesne, M. Loiselet, F. Lutton, I. D. Moore, Y. Martínez, E. Mogilevskiy, P. Naubereit, J. Piot, S. Raeder, S. Rothe, H. Savajols, S. Sels, V. Sonnenschein, J. C. Thomas, E. Traykov, C. Van Beveren, P. Van den Bergh, P. Van Duppen, K. Wendt, and A. Zadvornaya, Nature Communications 8, 14520 (2017).

${ }^{26}$ A. Zadvornaya, P. Creemers, K. Dockx, R. Ferrer, L. P. Gaffney, W. Gins, C. Granados, M. Huyse, Y. Kudryavtsev, M. Laatiaoui, E. Mogilevskiy, S. Raeder, S. Sels, P. Van Den Bergh, P. Van Duppen, M. Verlinde, E. Verstraelen, M. Nabuurs, D. Reynaerts, and P. Papadakis, Physical Review X 8, 41008 (2018).

${ }^{27}$ S. Sels, R. Ferrer, K. Dockx, C. Granados Buitrago, M. Huyse, Y. Kudryavtsev, S. Kraemer, S. Raeder, P. Van Den Bergh, P. Van Duppen, M. Verlinde, E. Verstraelen, and A. Zadvornaya, Nuclear Instruments and Methods in Physics Research, Section B: Beam Interactions with Materials and Atoms 463, 148 (2019).

${ }^{28}$ K. Dockx, T. E. Cocolios, R. Ferrer, C. Granados, S. Kraemer, Y. Kudryavtsev, S. Sels, P. Van den Bergh, P. Van Duppen, M. Verlinde, E. Verstraelen, and A. Zadvornaya, Nuclear Instruments and Methods in Physics Research, Section B: Beam Interactions with Materials and Atoms 463, 297 (2019).

${ }^{29}$ M. Verlinde, K. Dockx, S. Geldhof, K. König, D. Studer, T. Cocolios, R. de Groote, R. Ferrer, Y. Kudryavtsev, T. Kieck, I. Moore, W. Nörtershäuser, S. Raeder, P. Van den Bergh, P. Van Duppen, and K. Wendt, Applied Physics B 126, 85 (2020).

${ }^{30}$ R. P. De Groote, M. Verlinde, V. Sonnenschein, K. T. Flanagan, I. Moore, and G. Neyens, Physical Review A 95, 032502 (2017). ${ }^{31}$ M. Verlinde, Ph.D. thesis, KU Leuven (2020).

${ }^{32}$ K. Du, D. Li, P. Shi, S. Fu, C. R. Haas, X. Liu, B. Qi, J. Chen, and A. Schell, in ICALEO 2009 - 28th International Congress on Applications of Lasers and Electro-Optics, Congress Proceedings (2009) pp. 861-867.

${ }^{33}$ C. Granados et al., To be published.

${ }^{34}$ W. Gins, R. P. de Groote, M. L. Bissell, C. Granados Buitrago, R. Ferrer, K. M. Lynch, G. Neyens, and S. Sels, Computer Physics Communications 222, 286 (2018). 


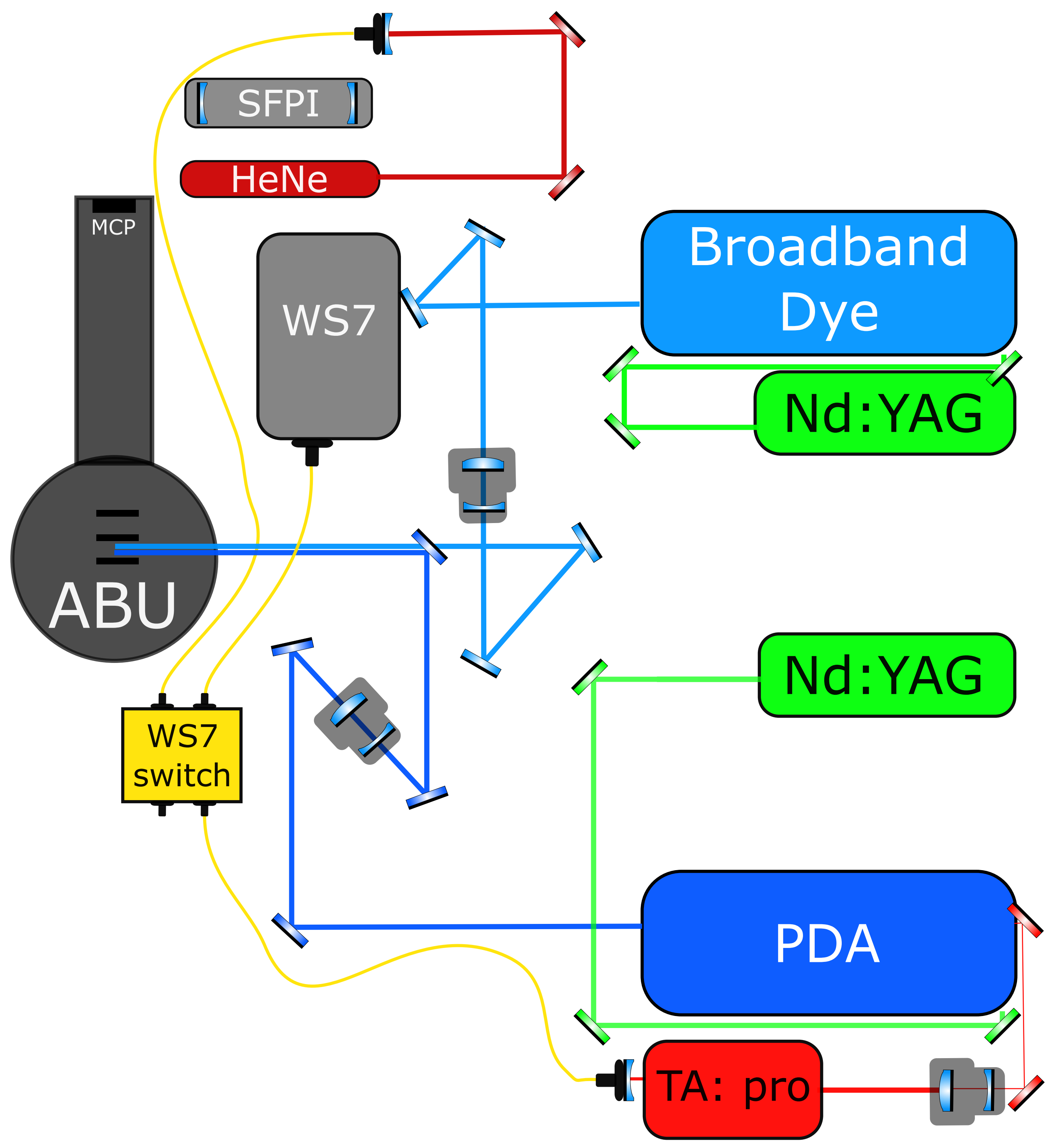




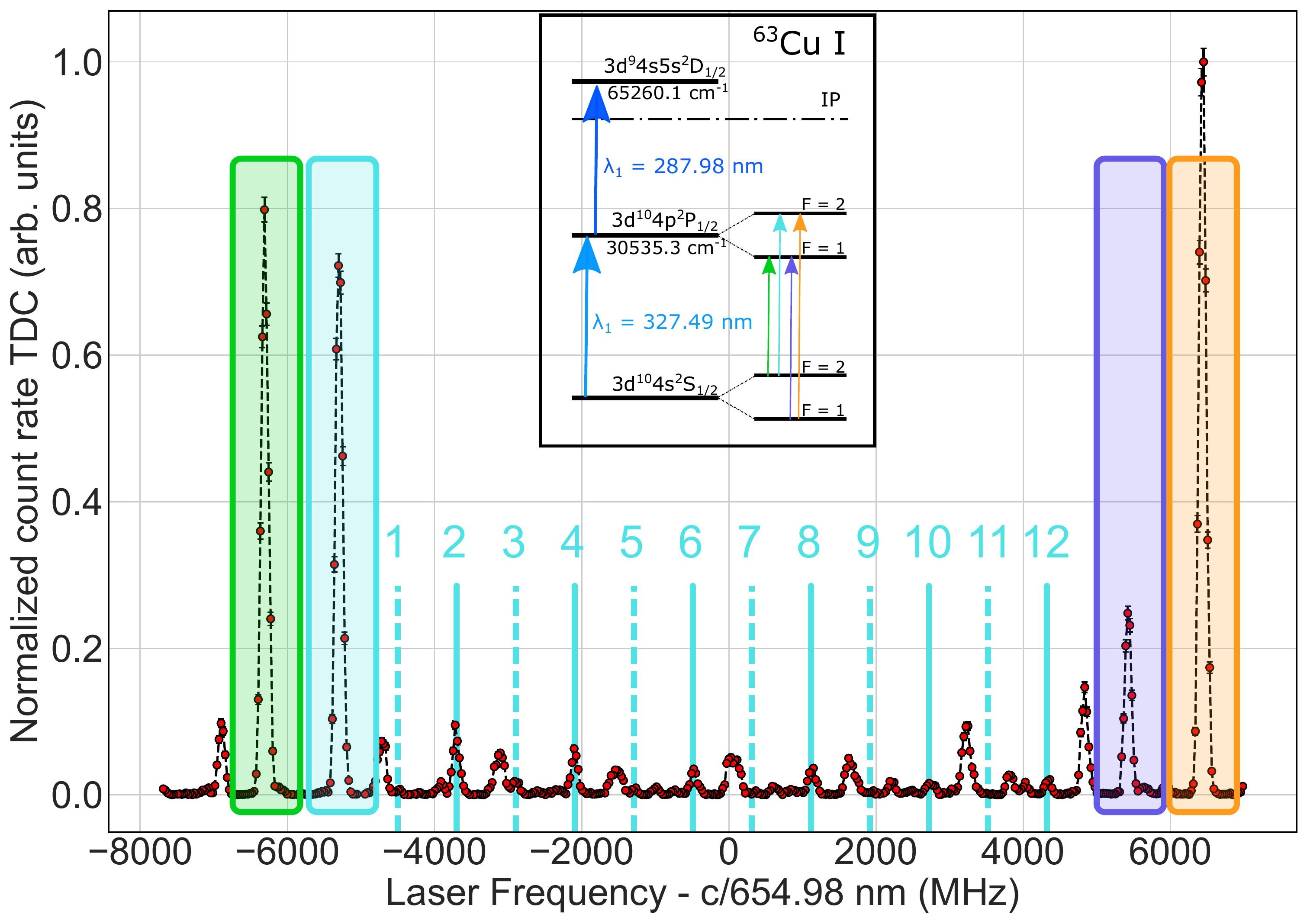




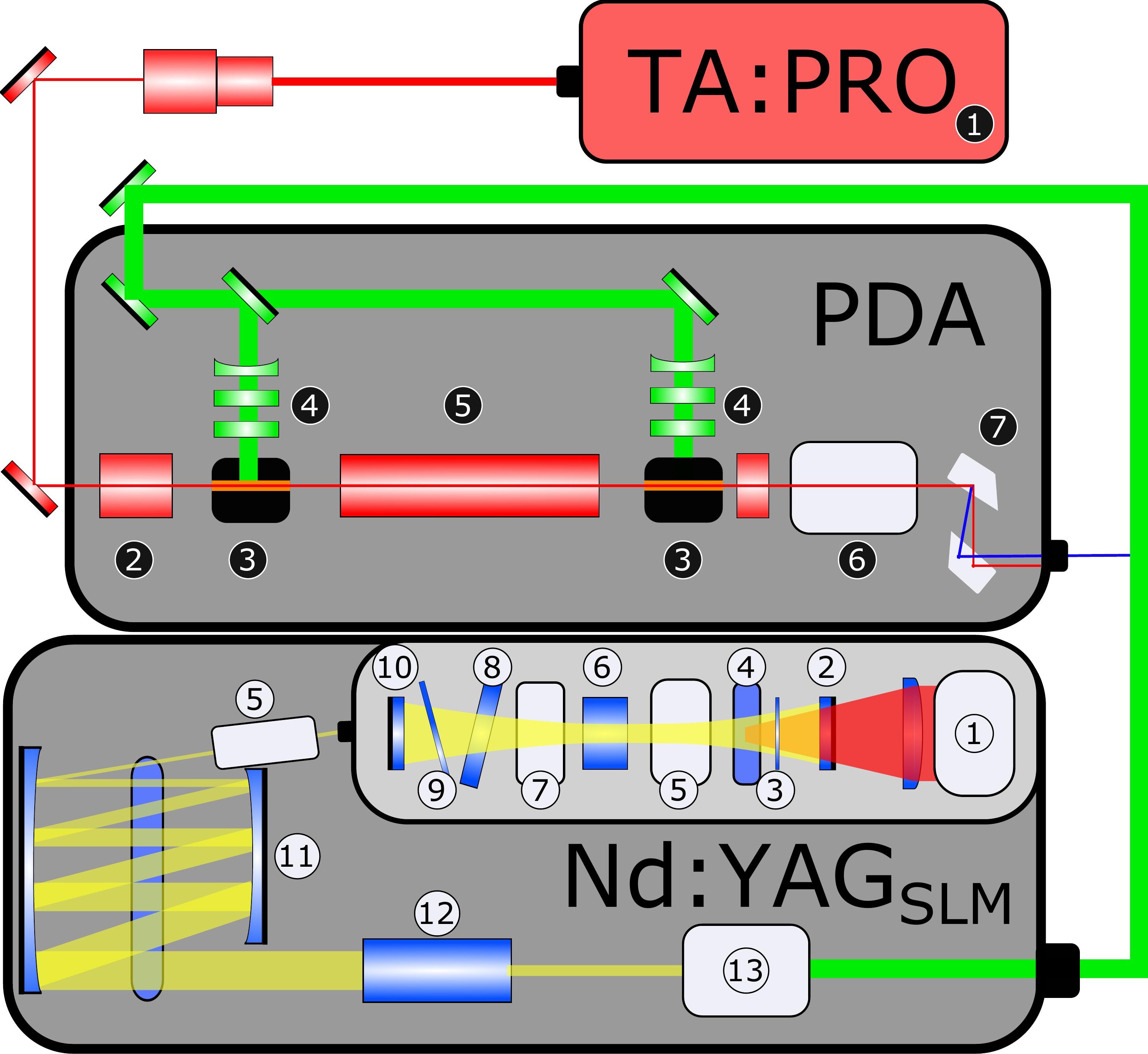




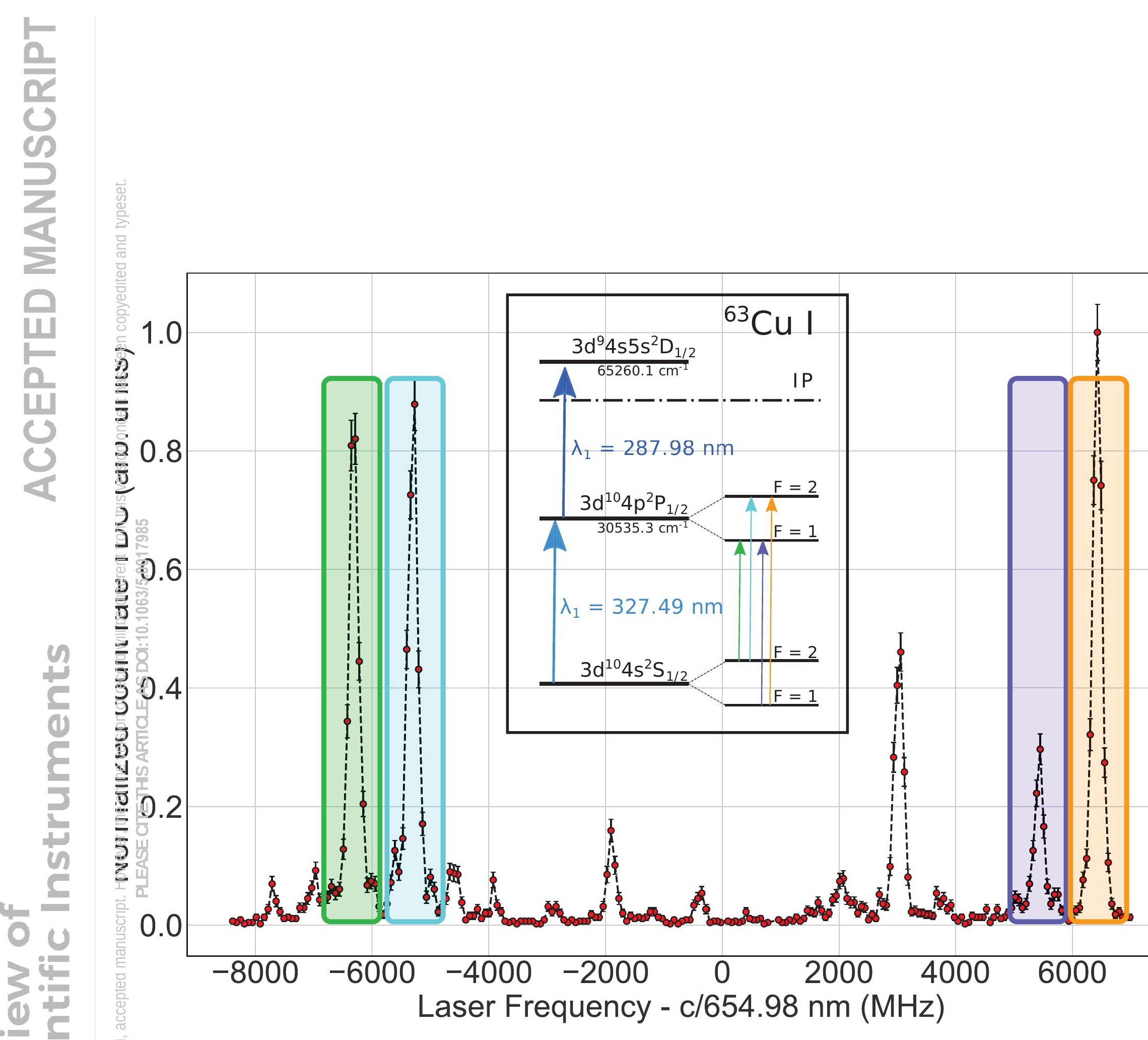




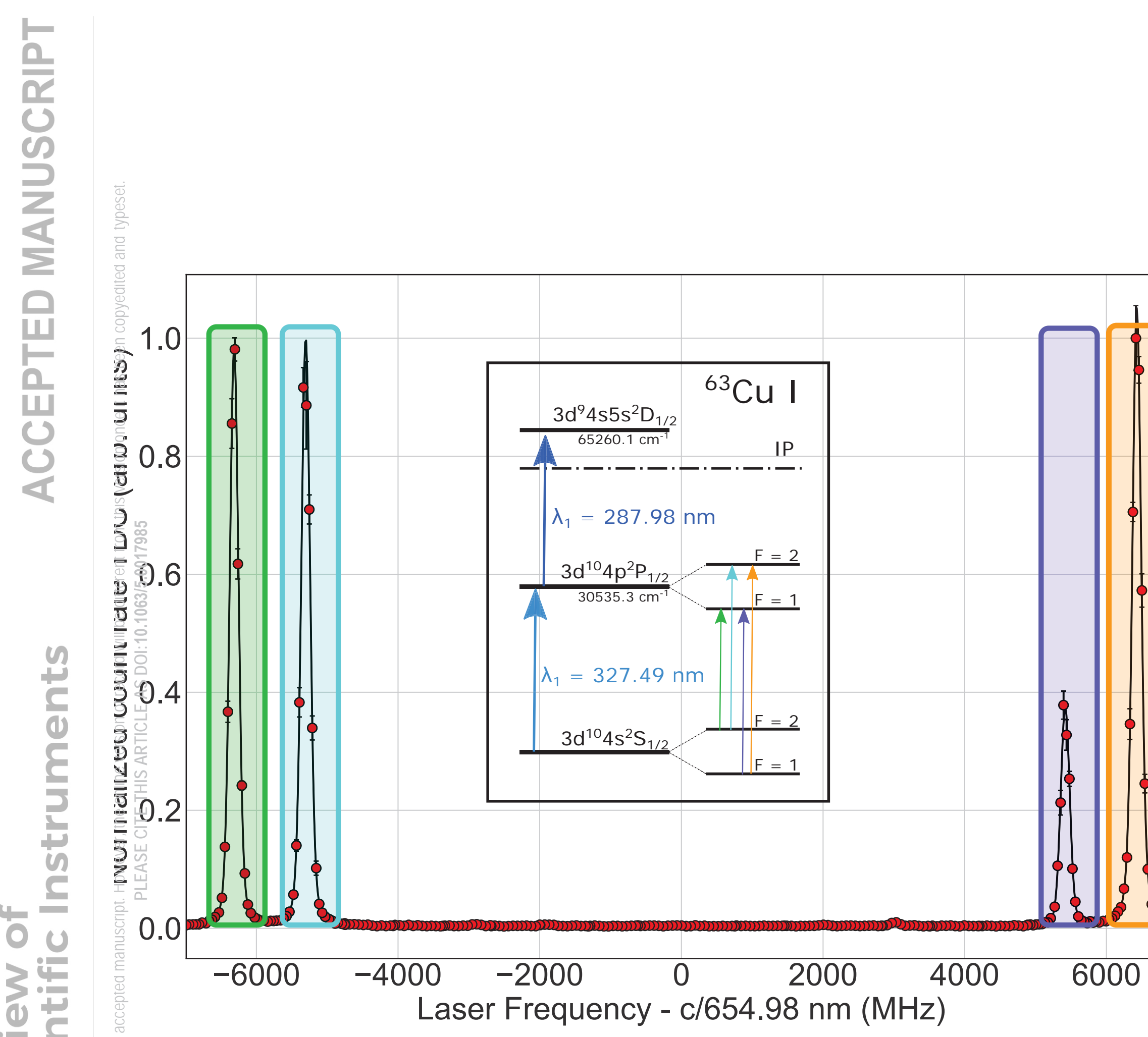

